\title{
Detección de la Presencia del Ácaro (Aculops lycopersici) Causante del Bronceamiento del Tomate (Lycopersicon esculentum) en El Salvador, AMÉRICA CENTRAL ${ }^{1}$
}

\author{
Leopoldo Serrano Cervantes ${ }^{1}$
}

\section{COMPENDIO}

En el mes de octubre de 1989, fueron enviadas preparaciones microscópicas de especimenes de ácaros con síntomas de bronceamiento y muerte de plantas jóvenes y adultas de tomate, procedentes de varias localidades de El Salvador, el acarólogo costarricense Ing. Ronald Ochoa, asociado a la Red de Diagnóstico del Centro Agronómico Tropical de Investigación y Enseñanza (CATIE), con sede en Turrialba, Costa Rica. El examen microscópico del material confirmó la identificación taxomónica como Aculops lycopersici (Acariformes:Eriophyldae), tal como se sospechaba desde la observación y colecta de muestras desde el mes de mayo de 1988. Un breve resumen de la literatura y una comunicación telefónica con dicho especialista que identificó el material, permite apreciar que esta especie que por primera vez se registra formalmente para El Salvador, constituye una importante plaga potencial que actualmente tiende a expandir su importancia en el trópico centroamericano. Se anotan algunas características de los hábitos y apariencias de esta especie con base en observaciones personales y a pequeños experimentos, así como a revisión de alguna literatura relacionada con este tema.

Palabras claves adicionales: plagas, hábitos del insecto.

\section{INTRODUCCION}

El cultivo del tomate en El Salvador, es una hortaliza de prominente lugar entre las hortalizas de mayor demanda de todos los hogares y a lo largo de todo el año. Por tratarse de una hortaliza que en muchos casos se consume en fresco, es deseable que en su cultivo se use lo mínimo posible de pesticidas contra los organismos que se constituyan en limitantes de su rendimiento, a fin de minimizar por un lado el nivel de posibles residuos tóxicos en el futuro; por otro lado la posibilidad de incremento poblacional de organismos fitófagos, actualmente como plagas potenciales del tomate o quizás en un nivel aún más bajo, llevándolos a niveles de abundancia económicamente peligrosas para el cultivo.

En este cultivo dentro del conjunto total de los riesgos de tipo fitosanitario, por lo general los principales son las enfermedades, aunque los daños debidos a artrópodos en determinadas situaciones pueden ser igualmente serios, algunos por sus daños como transmisores de enfermedades y otras por sus daños físico mecánicos entre los cuales algunas especies producen efectos tóxicos especiales que matan a la planta totalmente.

En muchos casos entre las plagas artrópodos existen especies pequeñas poco conocidas, como los ácaros que parecen proliferar en épocas secas; y entre las cuales hay es-

\begin{abstract}
3
In October 1989, microscopic specimens of (acaros) with symptoms of browning and plant mortality in young and older tomato plants from various locations in El Salvador were sent tó the entomologist Ing. Ronald Ochoa from Costa Rica. lng. Ronald Ochoa is associated with the Diagnostics Network of the Centro Agronomico Tropical de Investigation y Enseñanza (CATIE) based in Turrialba, Costa Rica. Microscopic examination of this material confirmed the taxonomic identification as Aculops lycopersici (Acariformes: Eriophyidae), suspected since May 1988 with the first observation and collection of samples. A brief review of literature and a phone conversation with the specialist who identified the material, permits reporting for the first time of the presence of this species in El Salvador. This finding is a warning about this pest which could be a potential problem in the tropics of Central America. This paper discusses personal observations of some of the characteristics, habits and appearances of this species, small experiments, and it includes a review of literature pertinent to this subject
\end{abstract}

pecies como los de la familia Eriophyidae que basta recientemente se han observado que están adquiriendo importancia cada vez mayor en algunos lugares de Centro América, como Costa Rica y El Salvador.

Este pequeño artículo pretende comunicar formalmente la detección de la especie eriofida Aculops lycopersici como una nueva plaga potencial para el cultivo del tomate en El Salvador; la cual hasta hoy no ha sido registrada; pero que se considera que en el corto plazo puede constituir suficiente motivo para desarrollar investigaciones orientadas al entendimiento de su biología, ecología y altenativas de manejo más apropiadas, dado que su efecto principal para el cultivo es la muerte rápida de la planta a cualquier edad. Adicionalmente se pretende ofrecer una revisión resumida de literatura científica relativa a su importancia y bioecología; tomando en consideración la insuficiente disponibilidad de ésta en las bibliotecas agrícolas de El Salvador, y aprovechando la gentil cooperación de la Red de Diagnóstico del

1 Trabajo preparado para ser presentado en la XXXVI Reunión Anual del Programa Cooperativo CentroamericanodeCultivos Alimenticios, PCCMCA; 26-30 marzo 1990, San Salvador, El Salvador, C.A. Aculops lycopersici (Massee 1937) (Acariformes:Eriophydae).

${ }^{2}$ Ing. Agr. Profesor de Entomología, Depto. de Protección Vegetal, Facultad de Ciencias Agronómicas. Universidad de El Salvador.

${ }^{3}$ El abstract es traducción del compendio.

Publicado en Agronomía Mesoamericana, Vol. 2 (1991). 
Cuadro 1 Procedencia, hospedero, y colectores del material biológico estudiado.

\begin{tabular}{|c|c|c|c|c|c|}
\hline $\begin{array}{c}\text { Caso } \\
\text { Observado }\end{array}$ & Hospedero & Localidad (procedencia) & Condición hospedero & Fecha & Colector \\
\hline $1 *$ & Tomate & $\begin{array}{l}\text { Opico, La Libertad } \\
(505 \mathrm{msnm})\end{array}$ & $\begin{array}{l}\text { Plántulas de almácigo } \\
\text { var. Santa Cruz Kada }\end{array}$ & Mayo 3, 1989 & $\begin{array}{l}\text { Víctor Manuel Miranda }{ }^{1} \\
\text { y Reina de Serrano }{ }^{2}\end{array}$ \\
\hline $2 * *$ & $\begin{array}{l}\text { Hierba } \\
\text { Mora }\end{array}$ & $"$ & $\begin{array}{l}\text { Plantas silvestre vecinas } \\
\text { al almácigo de tomate }\end{array}$ & Mayo 6, 1988 & $"$ \\
\hline 3 & $"$ & $\begin{array}{l}\text { Ciudad Universitaria, } \\
\text { San Salvador (700 msnm) }\end{array}$ & $\begin{array}{l}\text { Plantas silvestre } \\
\text { silvéstres }\end{array}$ & Mayo, 1988 & Leopoldo Serrano ${ }^{3}$ \\
\hline 4 & $"$ & $"$ & $"$ & Junio 1,1988 & $\begin{array}{l}\text { Leopoldo Serrano } \\
\text { Adán Hernández }{ }^{2}\end{array}$ \\
\hline $5 * *$ & $"$ & $\begin{array}{l}\text { Col. Jardinera de Cuscatlán, } \\
\text { La Libertad. ( } 860 \text { msnm) }\end{array}$ & $"$ & Junio, 1988 & Leopoldo Serrano \\
\hline $6 * *$ & Tomate & $\begin{array}{l}\text { Cantón Chaltipa, Santiago } \\
\text { Tezacuangos, San Salvador } \\
\text { (Rivera sur occ. Lago } \\
\text { Ilopango) (450 msnm) }\end{array}$ & $\begin{array}{l}\text { Plantas adultas y } \\
\text { senecentes, var. } \\
\text { Sta. Cruz } \\
\text { cultivada con tutores }\end{array}$ & Oct. 30,1988 & $\begin{array}{l}\text { Leopoldo Serrano } \\
\text { Reina de Serrano } \\
\text { Adán Hernandez } \\
\text { Mario A. Pérez Asencio }{ }^{4}\end{array}$ \\
\hline 7 & Tomate & $\begin{array}{l}\text { Col. Libertad } \\
\text { San Salvador } \\
(700 \mathrm{msnm})\end{array}$ & $\begin{array}{l}\text { Plantas adultas de } \\
\text { la variedad } \\
\text { Homestead - } 24\end{array}$ & Julio 4,1989 & $\begin{array}{l}\text { Azalea Herrera }{ }^{5} \\
\text { Reina de Serrano }\end{array}$ \\
\hline 8 & “" & $\begin{array}{l}\text { Ciudad Universitaria } \\
\text { Depto. S. S. (700 msnm) }\end{array}$ & $\begin{array}{l}\text { Plantas adultas de la } \\
\text { variedad U.C. } 82\end{array}$ & Julio 28,1989 & $\begin{array}{l}\text { Leopoldo Serrano } \\
\text { Heriberto Flores }{ }^{5}\end{array}$ \\
\hline
\end{tabular}

\footnotetext{
* Esta muestra original fue llevada al laboratorio del Centro Nacional de Tecnología Agrícola por el agricultor Víctor Manuel Miranda, en fecha 03 de mayo de 1988, a consulta por sospechas de padecer de alguna enfermedad. Examen en la muestra en tal laboratorio reveló la presencia de eriofidos que fueron enviadas al laboratorio del Departamento de Protección Vegetal de la Facultad de Ciencias Agronómicas de la Universidad de El Salvador, en donde se hicieron preparaciones microscópicas que aún se conservan a la fecha allí. Estas preparaciones y la observación de los síntomas en el hospedero conocido dieron origen a la sospecha de la presencia de un ácaro relacionado con el género Aculops de la familia Eriophyidae y ese diagnóstico preliminar fue sugerido al laboratorio del Centro Nacional de Tecnología Agrícola, a fin de poder dar una respuesta al agricultor. Posteriormente el agricultor llevó muestras de "hierba mora" con síntomas y ácaros similares.

** De estas muestras no se hicieron preparaciones microscópicas sino que únicamente se hizo un reconocimiento de síntomas del daño característico y de la presencia de los ácaros eriofidos.

${ }^{1}$ Agricultor cultivador de tomate

2 Técnicos del Departamento de Protección Vegetal Integrada del Centro Nacional de Tecnología Agrícola

3 Docente del Departamento de Protección Vegetal de la Facultad de Ciencias Agron6micas de la Universidad de El Salvador

${ }^{4}$ Estudiante de la Facultad de Ciencias Agronómicas de la Universidad de El Salvador.

5 Estudiantes tesistas del Departamento de Biología de la Facultad de Ciencias y Humanidades de El Salvador.
}

Centro Agronómico Tropical de Investigación y Enseñanza (CATIE), a través de la persona del Ing. M. Sc Ronald Ochoa, acarólogo con sede en Costa Rica.

\section{ANTECEDENTES}

En el año de 1988 se observaron casos de broncearniento y muerte de plantitas de almácigo y también plantas adultas de tomate, así como de bronceado, amarillamiento y defoliación de plantas de "hierba mora" (Solanum nigrum) localizadas como plantas no cultivadas en este último caso. Estos casos fueron observados en períodos secos dentro de los primeros meses de la época lluviosa, en ambientes rurales y urbanos.
En el año de 1989 se observaron síntomas similares en plantas de tomate adultas en el ambiente de una casa urbana y también dentro de un invernadero en el mes de julio en ambos casos. Estos casos presentaron ácaros amarillos de pequeño tamaño, patas cortas, aspecto corporal alargado y de coloración amarilla en diversas colonias localizadas principalmente a lo largo de las venas de las hojas y en las axilas de éstas y otros tejidos de crecimiento terminal de las plantas. Comúnmente los ácaros se encontraran sobre el haz de las hojas, completamente expuestas a la luz solar.

El material biológico estudiado procede de diferemes localidades y se debe a la colaboración de varias personas recolectoras, todo lo cual se resume en el cuadro 1 . 


\section{REVISION DE LITERATURA}

La especie Aculops lycopersici (Massee) fue descrita en 1937, a partir de especímenes procedentes del continente australiano, considerándose casi cosmopolita; en la actualidad por lo menos dentro del rango de las latitudes $60^{\circ}$ norte y $60^{\circ}$ sur (Jeppson et al., 1975; Magalhares, 1982; Perring y Parrar, 1986). En América, ocurre en los Estados Unidos (California); en Venezuela (Doreste, 1984; Gallo et al., 1978; Jeppson et al., Magalhares, 1982) y para el área de América Central se conoce su ocurrencia en Costa Rica (Salas et al., 1982) y también El Salvador tal como se informa en el presente trabajo, gracias a la confirmación taxonómica autorizada del Ing. Ronald Ochoa, Acarólogo asociado al Centro Agronómico Tropical de Investigación y Enseñanza (CATIE) con sede en Turrialba, Costa Rica, el 10 de octubre de 1989.

Esta especie se conoció por primera vez en América del Sur en 1962, citándose por Rossi en Argentina, luego Fletchman y Aranda la citan en 1970 en Brasil y Cermeli et al. (1982) la mencionan en Venezuela. En 1963 este ácaro era conocido en 28 países y el año de 1986 ocurría en un total de 37 países (Perring y Farrar, 1986).

Se conoce que este ácaro ataca al tomate en forma tan severa que provoca finalmente la muerte de la planta; pudiendo darse ataques de esta plaga bajo condiciones de invernadero (Berlinger et al., 1982). Son atacadas además otras plantas de la familia Solanaceae, tales como papa, berenjena, tabaco, chile, petunio y hierba mora; hospederas en la cuales, o bien las poblaciones no son tan numerosas, o bien no provocan efectos drásticos como en el caso del tomate, aunque

Fuertes infestaciones en algunas de ellas (papa, petunia, tabaco y chile) pueden derivar en daños y en el caso especial de la berenjena se presenta una gran capacidad de sobre vivencia o tolerancia de la planta. La hierba mora (Solanum nigrum $\mathrm{L}=S$. nudiflorum Sendt $=S$. americanum Mill $=S$. oleraceum Dun) es considerada como un importante hospedero silvestre del ácaro. Esta plaga ha sido localizada en plantas de la familia Canvolvulaceae, tales como Convolvulus arvensis e Ipomoea purpurea (Chousy, 1976; Doreste, 1984; Gallo et al. 1984; Gómez y Rivera, 1987; Jeppson et al., 1975; Magalhares, 11982).

En 1940, Keifer, citado por Perring, (1986) afirmaba que el ácaro podría ser encontrado sobre plantas Convolvulus, pero sin ocurrir actividad de reproducción; pero en 1962, Rice Strang citados también por el autor ya referido (Perring, 986), encontraron que la reproducción en tal hospedero sí ocurría y producía abundante población en la planta. Los ismos autores también pudieron encontrar que no pudieron tener evidencias de reproducción del ácaro sobre plantas de Nicotiana (tabaco), a pesar de que en 1956 y 1960 Smith y Saunders habían mencionado a esta especie de planta como huésped de Aculops lycopersici.

Existen autores citados por Royalty y Perring, (1987) que interpreten la interacción planta-herbívoro para el caso de esta plaga, come excepcional dentro de la superfamilia Eriophyoidea considerando que la infestación sobre la planta no causa solo deformaciones sino la muerte misma de la planta. En apoyo a esta consideración, en 1943 Baileyy Keifer, yen 1976 Hilstop y Jeppson, citados por los mismos autores ya referidos (Royalty y Perring, 1987) sostienen que existen evidencias que sugieren que el tomate no es el hospedero evolucionario para Aculops lycopersici, ya que este hospedero provoca el fenómeno llamado "Estimulación Solanum ", el cuál consiste en una reproducción veloz que también produce rápidamente la muerte de la planta, lo que se interpreta como una situación desventajosa para un organismo parasitario. Este fenómeno sin embargo no se produce en Petunia spp, ni en Convolvulus arvensis también colonizadas por esta especie de ácaro.

Algunos autores se refieren a este ácaro como ácaro de la mancha color canela del tomate (Jeppson et al., 1975), ácaro del bronceamiento del tomate (Gallo et al., 1978; Magalhares, 1982), ácaro tostador del tomate (Cermeli et al., 1982; De Olivera et al., 1982), microácaro a ácaro del bronceado y la acronecrosis del tomate (Gallo et al., 1978). En México, el daño ocasionado por la infestación de éste ácaro es designado con el término "canelilla" (Ochoa, R. 1989), Comunicación personal.

El ácaro causante del bronceamiento del tomate, ha sido mencionado con un conjunto de nombres científicos que de acuerdo a Cermeli (1982) y Perring, (1986), actualmente constituyen sinónimos del nombre actualmente usado: $\mathrm{Acu}$ lops lycopersici. Tales sinónimos son los siguientes:

Phyllocoptes lycopersici Tyron 1917

Phyllocoptes lycopersici Massee 1937

Phyllocoptes destructor Keifer 1940

Phyllocoptes lycopersici Keifer 1940

Vasates destructor Keifer 1946

Vasates lycopersici Lamb 1953 y Anderson 1954

Aculus lycopersici Keifer 1959

Aculops lycopersici Keifer 1966, Fletchmann y Aranda1970

Perring (1986) cita que Roffs infornló en 1982 en Florida, acerca de un daño observado en plantas de tomate, producido por un pequeño ácaro de cuatro patas. Tal observación, aunque no concuerda con los síntomas que hoy día se consideran típicos para la infestación por Aculops lycopersici, pareciera ser la referencia más antigua de problemas de ácaros eriófidos, en el cultivo del tomate. Este mismo autor presentó una serie de comentarios relativos a cmnbios en la nomenclatura de esta especie. 
De acuerdo a Perring (1982) la descripción original de las hembras de esta especie se debe a Massee en 1937, quien midió en ellas, longitudes de 200 micras y anchuras de 40 micras. Los primeros esquemas de todos los estadíos vitales, las garras plumosas, el patrón de marcas en el escudo cefalotorácico y lagenitalia de ambos sexos se deben a Bailey y Keiferen 1943.

Según indica Keifer et al., (1982) la morfología del escudo dorsal es característica para este ácaro, lo cual j unto con otros rasgos corporales permite reconocer la especie. Este autor ha ilustrado en forma muy sintética y de fácil entendimiento; tales rasgos.

Kay (1986) cita que los individuos adultos de esta especie miden desde 150 micras hasta 200 micras de longitud y alrededor de 50 micras de ancho.

Según Cermeli et al., (1982) el diámetro de los huevos es alrededor de las 50 micras. Royalty y Perring, (1989) anotan que las especies de ácaros Eriophyldae, que causan bronceamiento presentan cortos estilete s en su aparato bucal: 7 a 20 micras en contraste con los estiletes más largos que presentan los ácaros Tetranychidae 130 micras, tal como se deduce de los trabajos de McCoy y Albrigo en 1975, y Jeppson en 1975, citados por tales autores. Jeppson et al., (1975) describen con detalles morfológicos precisos la caracterización taxonómica de la especie Aculops lycopersici y algunos de los rasgos más generales de tal descripción son los siguientes: Se trata de una especie estructuralmente diferente de otras especies congéneres. Su apariencia es fusiforme y algo robusta y su color amarillo naranja. Las hembras miden desde 150 hasta 180 micras de largo. Las garras plumosas de las patas presentan cuatro rayos. El abdomen tiene alrededor de 27 terguitos y 60 estemetos. Otros rasgos más precisos de tipo morfológico taxonómico también son citados por estos autores y deben ser consultados para propósitos de exámen taxonómico de preparaciones microscópicas.

Magalhaes Bastos, (1982) resume los síntomas principales del daño debido a Aculops lycopersici en plantas de tomate, así: perior

a) Bronceamiento de hojas y pedúnculos en su parte su-

b) Caída de hojas

c) Muerte de la planta.

Jeppson et al., (1975) hacen una resefia más pormenorizada de los síntomas del daño, la cual es también descrita en forma similar por Doreste, (1984). De tales autores se extraen las siguientes anotaciones en relación a la interacción planta de tomate - ácaro del bronceamiento: "Las hojas se toman de color café y su textura se asemeja a la del papel; pero no se marchitan pudiendo desprenderse algunas de ellas antes de cambiar su coloración. Los tallos infestados adquieren una apariencia bronceada o coloración canela, y con frecuencia se agrietan longitudinalmente y mueren. Los frutos generalmente no adquieren la apariencia bronceada; pero pueden sufrir quemaduras de sol cuando ocurre caída de hojas" (Jeppson et al., 1975; Doreste, 1984). Royalty y Perrillg, (1986) han estudiado los aspectos fisiológicos del daño de esta plaga en as hojas en relación al bronceamiento y deformación por encrespamiento; descartando la acción de alguna toxina. De Oliveiro et al. (1982) citan que los síntomas del ataque de esta plaga, se observan 10 días después del inicio de la infestación. Kay (1986) explica que en las plantas infestadas se reduce el número de frutos cuajados reduciéndose en consecuencia el rendimiento y los frutos quedan en la planta y resultan infestados. adquieren una apariencia no atractiva debido a manchas en forma de halo blanco o amarillento, lo que se presenta tanto en frutas verdes como en frutas que ya tienen alguna coloración de madurez. De Oliveira et al. (1982) afiaden que en relación a los frutos de tomate; el ácaro prefiere habitar la superficie del fruto, próxima al pedúnculo en donde produce pequefias rajaduras.

\section{METODOLOGIA}

Los ácaros encontrados asociados a plantas de tomate con síntomas de bronceamiento en hojas o tallos, los cuales finalmente se secaron y murieron; fueron colectados y se dispusieron en preparaciones para observación microscópica empleando esencialmente como medio de montaje el de Hoyer, antecediénlo por inmersión de los especfmenes en lactofenol en algunas ocasiones o de preservación tem poral en alcohol etílico al $60 \%$ en otras ocasiones. En ambos casos las muestras de ácaros fueron desprendidos masivamente del substrato vegetal (por lo general, follaje) infestado; mediante inmersión en agua caliente por breves momentos. En el caso deácaros procedentes del hospedero silvestre: "hierba mora" (Solanum nigrum) el procedimiento de manejo de la muestra fue similar al ya descrito en relación al material del tomate.

La clasificación y el sellado de las preparaciones microscópicas se aceleraron mediante exposición a vapor de agua durante 4 ó 5 minutos.

\section{RESULTADOS Y DISCUSIÓN}

Los ácaros causantes de bronceamiento del tomate por lo general se localizaron en el haz de las hojas y con más abundancia a lo largo de las venas; tanto en el caso del hospedero tomate como en el caso del hospedero silvestre hierba mora. La coloración de estos ácaros es amarillo intenso. El síntoma de bronceamiento fue común en tallos y pecíolos foliares además de las hojas, siendo común la apariencia amarillenta de hojas de plantas afectadas por ácaro en el caso de "hierba mora". No se tuvo oportunidad de observar síntomas de daño en frutos porque en casi todas la ocasiones las plantas de tomate murieron antes de formar fruto.

En relación a los experimentos de infestación artificial pudo detectarse que al morir la hoja infestada usada como inóculo, los ácaros la abandonaron y se dispersaron por la planta sana y en menos de utla semana pudieron verse 
cambiar de coloración de verde nonnal a bronceado, en los tallos, iniciándose dicho efecto en las axilas de los pecíolos foliares. Las plantas de tomate así infestadas murieron completamente sin ocurrir desprendimiento de sus hojas.

La observación de la preparaciones microscópicas en el laboratorio del Departamento de Protección Vegetal de la Facultad de Ciencias Agronómicas de la Universidad de El Salvador, evidenció que los especímenes pertenecían a la familia Eriophyidae en base a la morfología típica y característica de estos organismos.

En relación a la observación de los hábitos de alimentación y producción de síntomas típicos del daño de este ácaro, se realizaron infestaciones artificiales a plantas sanas de tomate, partiendo de plantas de tomate o de hierba mora que presentaban infestación natural. Esto se realizó fijando hojas infestadas, al tallo de las plantas sanas mediante angostas cintas de papel engomado. Estos pequeños experimentos se realizaron en los laboratorios del Departamento de Protección Vegetal de la Facultad de Ciencias Agronómicas de la Universidad de El Salvador y también en una casa de habitación de la colonia Jardines de Cuscatlán, jurisdicción de Antiguo Cuscatlán. Departamento de la Libertad.

Las preparaciones microscópicas se aprovecharon para hacer dibujos de la morfología externa, comparar las dimensiones corporales de estos ácaros y tomar fotografías.

Aprovechando la experiencia de observación de síntomas en infestaciones controladas y también la observación morfológica de los ácaros, se analizó el ácaro a la luz de alguna literatura científica relacionada con ácaros asociados con el cultivo del tomate. Tal procedimiento condujo a la sospecha de que la especie en cuestión podría corresponder a Aculops lycopersici (Massee) lo cual y en tales ténninos fue expuesto al ingeniero Ronald Ochoa, acarólogo asociado a la Red de Diagnóstico del Centro Agronómico Tropical de Investigación y Enseñanza con sede en Turrialba, Costa Rica, mediante nota escrita dirigida a él con fecha octubre 09 de 1989, junto con varias preparaciones microscópicas para ser examinadas taxonómicamente y obtener de este especialista, la correcta identificación del ácaro en cuestión. Este procedimiento pennitió confmnar las sospechas de la identificación del ácaro como Aculops lycopersici; lo cual fue informado así por el taxónomo, el día 10 de octubre de 1989, en Costa Rica desde 1982 y que actualmente se sospecha también de su presencia en Guatemala.

La ubicación taxonómica de esta especie de ácaro se gún Krantz (1978) y Jeppson et al., (1975) es como sigue:

Phyllu:Arthropoda

Clase: Arachnida

Subclase: Acari

Orden: Acarifonnes

Suborden: Actinetida

Super Cohorte: Promatides
Cohorte: Eleutherengonina

Sub Cohorte: Raphignatbae

Super familia: Eriophyoidea

Familia: Eriophyidae

Subfamilia: PhyIlocoptinae

Sección: V

Género: Aculops Keifer et al., 1966

Especie: A. lycopersici Massee, 1937

La morfología externa de los especímenes de esta especie fue ilustrada por medio de esquemas dibujados con ayuda de un microscópio compuesto provisto de tubo para dibujo yen base a preparaciones microscópicas de especímenes obtenidos de colonias criadas en tomate o en hierba mora. Pudo detectarse que estos ácaros son susceptibles acierto grado de ser teñidos en su tegumento, por efecto de los colorantes Safranina y Verde de Malaquita.

El microscopio utilizado fue uno del tipo LEITZ-Laborlux- D provisto de objetivos 4x, 10x,40x y 100x y provisto de una escala para medición micrométrica previamente calibrada. Bajo tales condiciones se realizaron algunos dibujos con reglobro de sus dimensiones reales y de la magnificación aproximada alcanzada en cada dibujo. Las principales mediciones corporales fue la longitud (desde el extremo del enatbosoma, hasta el extremo caudal; sin tomar en cuenta longitud de patas, queliceros o setas caudales) y anchura generalmente medida como su valor máximo, lo que en la práctica por lo común se ubicó inmediatamente por detrás del escudo cefálico. Se registró en cada caso la posición del espécimen observado en las preparaciones microscópicas: lateral, dorsal, o ventral, lo cual en ocasiones puede influir en la apreciación correcta o más precisa de las dimensiones. Estas dimensiones se utilizaron para calcular la proporción longitud/anchura en cada espécimen.

El registro de los tamaños de los especímenes (con base en el largo y ancho principalmente) representa el examen de 98 ácaros de colonias criadas en tomate y 56 ácaros de colonias criadas en " hierba mora". Los datos se ordenaron de la mayor a la menor longitud por observarse mayor variabilidad en esta dimensión, que el ancho del cuerpo.

Además de la posición del espécimen sometido a medición, se considera que ésta puede ser afectada por el sexo, la gravidez o virginidad, o la edad misma del especímen; detectándose fácilmente en relación a las mediciones (tanto en trazos de la escala micrométrica como su equivalencia en micras), indi viduos relativamente largos o cortos, o bien; flacos o gordos. Se aprecia que el cuerpo del ácaro es más ensanchado dorsalmente que ven tralmen te, lo cual se deduce de la observación de la orientación de las hileras de microtubérculaciones y de las líneas interterguitos.

El comportamiento de los datos de medición de longitudes de los ácaros procedentes de plantas de tomate, mostraron un rango de 61.00 micras hasta 217.16 micras. Paralas mediciones de anchura, el rango fue desde 36.60 micras hasta 
73.20 micras. Atendiendo a la variación y frecuencia de los valores de longitud, puede considerarse que probablemente las longitudes del ordende 61, 73,85, 90 y quizás hasta 100 micrascorresponden a los primeros estadíos del ácaro. La relación longitud/ancho produjo valores desde 1.61 hasta 3.95.

El comportamiento de los datos de medición de longitud de los ácaros procedentes de plantas de hierba mora, mostraron un rango desde 46.36 micras hasta 185.44 micras. Para las mediciones de anchura, el rango fue desde 24.40 hasta 61.00 micras. Atendiendo a la variación y frecuencia de los valores de longitud, puede considerarse que probablemente las longitudes del orden de 46, 73, 85, 90, 97 y quizás hasta 100 micras, corresponden a los primeros estadíos del ácaro. La relación longitud/anchura produjo valores desde 1.60 hasta 3.85 .

Haciendo una comparación muy preliminar entre lo ácaros provenientes de los dos hospederos estudiados se puede proponer que en ambos casos la proporción longitud/anchura, parece comportarse en forma similar, pero en relación al tamaño de los especímenes, los rangos para longitudes y anchuras parecen sugerir que los especímenes provenientes de hierba mora pueden ser más pequeños que los que se crían en tomate. En relación al rango de longitudes de los especímenes provenientes de plantas de tomate (61.00-217.6 micras) se observa que difiere un poco del rango mencionado por otros autores (Jeppson et al., 1975; Perring y Farrar, 1986) especialmente para hembras adultas y que va desde 150 hasta 180 o 200 micras. Algo similar ocurre en relación al ancho del cuerpo, el cual en los especímenes criados de plantas de tomate va desde 36.60 hasta 73.20 micras y en el caso de algunos autores (Perring y Farrar, 1986; Kay, 1986) se señalan cifras de 40 o 50 micras. Todo ello hace pensar en que es posible que en determinado momento, las condiciones de tipo físico y/o biótico que forman parte del medio ambiente en donde se desarrolle esta plaga pueden influir en el tamaño corporal de la especie.

Con las mediciones realizadas no es de valor práctico calcular promedios de tamaño del ácaro porque esmuy probable que en las colonias estudiadas existan diferentes estadíos de desarrollo de la especie que aún no se conocen en su morfología diferencial para las condiciones de El Salvador.

Se presentan algunos dibujos correspondientes a especímenes de Aculops lycopersici, debiéndose hacer la advertencia: que algunos rasgos importantes del escudo dorsal, tales como varias líneas transversales en la banda periférica del Escudo o Placa Cefálica Dorsal; no se ilustran por no haberse contado con un microscopio de contraste de fases para poder detectarlas.

\section{CONCLUSIONES}

1. El organismo causal de fenómenos de bronceamiento del tomate, con consecuencias letales para la planta en El
Salvador, corresponde a una especie de ácaro de la familia Eriophyidae, cuya determinación taxonómica es Aculops lycopersici (Massee 1937).

2. Aculops lycopersici (Massee 1937) representa una nueva plaga potencial para el cultivo del tomate en El Salvador, cuya importancia puede incrementarse bajo condiciones de variedades susceptibles, condicionesclimáticas típicas de época seca, presencia de hospederos silvestres y tal vez un uso inadecuado de pesticidas en el cultivo.

3. Un hospedero silvestre común para esta especie de ácaro en El Salvador es la planta conocida como hierba mora (Solanum nigrum Sendt); por lo que el manejo apropiado de estas plantas puede representar una táctica de control cultural dentro de un posible manejo integrado del ácaro plaga.

4. Los individuos de las colonias del ácaro, estudiadas, alcanzan su tamaño mayor correspondiendo a longitudes de 217.16 micras y anchura de 73.20 micras en el caso del hospedero tomate ya longitudes de 185.44 micras y anchuras de 61.00 micras en el caso del hospedero hierba mora, siendo en ambos hospederos la misma relación longitud/anchura (Aproximadamente de 4).

\section{RECOMENDACIONES}

Con base a la información contenida en la literatura revisada en relación a la importancia de la especie Aculops lycopersici para el cultivo del tomate; y a lo reciente de su detección en El Salvador, se proponen las siguientes recomendaciones:

1. La importancia de este fitófago para el cultivo del tomate, justifica y demanda urgentemente el inicio de trabajos de investigación relacionados con el entendimiento de su bioecología a fin de fundamentar el manejo más racional posible de sus poblaciones plaga en las localidades del país donde sea detectada su presencia.

2. Este ácaro plaga, de acuerdo a la literatura es posible que se presente asociado con diversos enemigos naturales tal como refleja la literatura; siendo en su mayoría especies de ácaros depredadores los cuales pueden resultar muy valiosos como agentes de biocontrol tanto en hospederos silvestres como en hospederos cultivados, razón por la cual debe iniciarse su búsqueda en los diferentes ecosistemas del país.

3. Esta especie de ácaro plaga, de acuerdo a la literatura parece ser susceptible a varios productos químicos, lo cual sugeriría un fácil control a corto plazo, aunque ya existen algunos avisos de la precaución que debe tenerse en este tipo de control cuando se use unilateralmente; razón por la cual, para nuestro país deberán hacerse las consideraciones previas de tipo ecológico, fitofisiológico y económico, cuando el control químico de la plaga sea urgentemente 


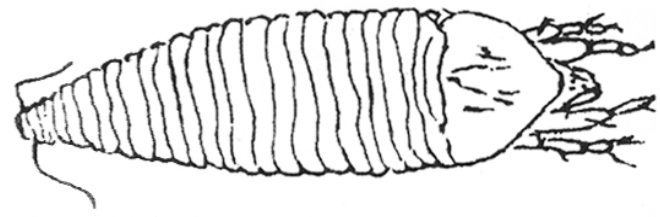

Figura 1 Vista Drosal

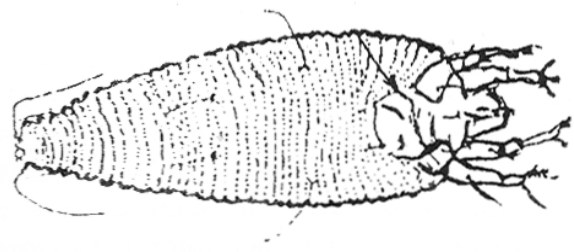

Figura 2 Vista ventral

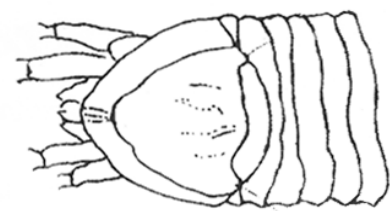

Figura 3 Vista dorsal

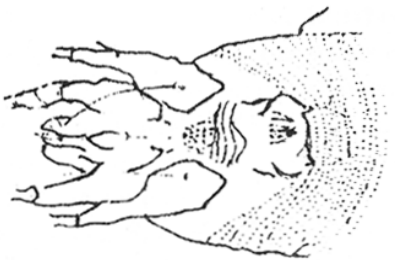

Figura 4 Vista ventral requerido, procurando en todo caso realizarlo bajo la filosofía de manejo integrado de este problema potencial.

4. Previniendo la ocurrencia de problemas serios por esta plaga a medida que se distribuya más ampliamente en el país, se considera conveniente iniciar estudios sobre evaluación de variedades en búsqueda de fuentes de tolerancia o de mayor resistencia a este ácaro en los cultivares de uso común actualmente en El Salvador, para disminuir la dependencia obligada al control unilateral de tipo químico para esta especie fitóloga del cultivo del tomate.

\section{BIBLIOGRAFÍA}

BERLINGER, M.J.; DAHAN. R.; and COHEN, S. 1982. Greenhouse tomate pests and their control in Israel. Proc. Working Group Integrated Control in Glasshouses. Darmstadt. June 26-29. 1982. Bull IOBCI/WPRS/1983/V/I3; 7- 11.

CERMEU, M.; DORESTE, S. E.; VAN BALEN. L. 1982. Aculops lycopersici (Massee 1937) (Acari:Eriophyidae) plaga del cultivo del tomate en Venezuela. Rev. Fac. Agron. (Maracay). XII (3-4): 227.234.

CHOUSSY, F. 1976. Flora Salvadoreña. Tomo II Colección Temachtiani 2a. Ed. Editorial Universitaria. Universidad de El Salvador. ,San Salvador. p. 77

DE OLIVERA, C.A.L.; ESCHTAPATI, D.; VELHO. D.; SPONCHIADO. 0.1. 1982. Daños cuantitativos causados pelo microácaro Aculops lycopersici (Massaee 1937) en cultura de tomatero. Ecosisterna (Brasil) Vol. 7. pp. 14-18.

DORESTE. S.E. 1984. Acarología. Instituto Interamericano de Cooperación para la Agricultura. Serie Investigación y Desarrollo No. 11. San José. Costa Rica. p. 61. 72, 76, 95. 204, 209, 219, 375.377.

GALLO, D. et al. 1978. Manual de Entomología Agrícola, Editora CARES. Edicao "CARES III". Sao Paulo. p. 441.
GOMEZ ARISTIZABAL, A.; RIVERA POSADA, H. 1987. Descripción de malezas en plantaciones de café. Centro Nacional de Investigaciones del Café: CERNICAFE. Carvajal S. A. Chinchiná, Colombia. p. 402

JEPPSON. L. R.; KEIFER. H. B.; BAKER. E.W. 1975. Mites injurious to economic plants. University of California Press. pp. 359. 383. 502. $507,508,551,555$

KAY, I. R. 1986. Tomato russet mite: a serious pest of tomatoes. Queensland Agricultural JournaI112(5): 231.232.

KEIFER. B.H.; BAKER. E.W.; KONO. T.; DELFINADO. M.; STYER, W.E 1982. An illustrated guide to plant abnormalities caused by eriophyid mites in Nort America. U.S.D.A. Handbook No. 573. pp.130-131.

KRANTZ. G.W. 1978. A manual of Acarology. 2nd. ed. Oregon State University Book Stores. Corvaelis. Oregon.pp. 100101.

MAGALHAES BASTOS. J. A. 1982. Principais pragasdas culturas seus controles. 2a. Ed. rev. Livraria Nobel: S.A. Sao Paulo. pp. 209.210.

PERRING.T.M.; FARRAR. Ch. A. 1986. Hístorical perspectue and current world status ofthe Tomato Russet Mite (Acari: Eriophyidae). Ent. Soco of Am. Misc. ubl. No. 6319 p,

ROYALTY. R.N.; PERRING. T.M. 1987. Comparative Toxicity of acaricides to Aculops lycopersici and Homeopronematus anconal (Acari:Eriophyidae. Tydeidae) J. of Econ. Ent. 80(2): 348-351.

ROYAL TY, R.N.; PERRING, T.M. 1988. Morphalogical Analysis of damage to tomato leaflet by tomato Russet Mite (Acari: Eriophyidae) J. of Econ. Ent. 81 (3): 816.820. 16.

ROYALTY. R.N.: PERRING. T.M. 1989. Production in photosynt of tomate leaflets caused by Tomato Russet Mite (Acari: Eriophyidael Eviren. En!. 18 (2): 257-260.

SALAS F.., L.A. 1982. Aculops lycopersici (Massee) (Eriophyidae: Acari) una nueva plaga deltomate en Costa Rica. V. Congreso Agronómico Nacional. Julio 1982. San José C.R. VI. i. Resúmenes p. 44. 